\title{
Mathematical Support for Automated Design Systems for Passive Optical Networks Based on the $\beta$-parametric Approximation Formula
}

\author{
Oleksiy Nedashkivskiy ${ }^{1}$, Yevhen Havrylko ${ }^{2}$, Bohdan Zhurakovskyi ${ }^{3}$, Juliy Boiko $^{4}$ \\ ${ }^{1}$ Professor, Department of Automation of Design of Energy Processes and Systems, National Technical University \\ of Ukraine "Igor Sikorsky Kyiv Polytechnic Institute", Kyiv, Ukraine. al_1@ ua.fm \\ ${ }^{2}$ Professor, Department of Automation of Design of Energy Processes and Systems, National Technical University \\ of Ukraine "Igor Sikorsky Kyiv Polytechnic Institute", Kyiv, Ukraine. gev.1964@ukr.net \\ ${ }^{3}$ Professor, Department of Technical Cybernetics, National Technical University of Ukraine "Igor Sikorsky Kyiv \\ Polytechnic Institute", Kyiv, Ukraine. bogdan68@ukr.net \\ ${ }^{4}$ Professor, Department of Telecommunications and Radio Engineering, Khmelnytskyi National University, \\ Khmelnytskyi, Ukraine. boiko_julius@ukr.net
}

\begin{abstract}
The paper considers the models of passive optical networks, namely the balancing of network branches. At the same time, models of passive optical splitters with one input and two or three outputs were used, which is based on a $\beta$-parametric approximation formula that takes into account the type of splitter and two accuracy classes. On the basis of the obtained equations and the performed calculations, a unified universal mathematical model is proposed that allows balancing splitters with any number of output ports in automatic design systems.
\end{abstract}

Key words : Automatic design system, balancing, passive optical network, software.

\section{INTRODUCTION}

Current advances in fiber optic technology, in particular the development of Passive Optical Networks (PON), offer broad opportunities for broadband access to multiservice networks and other telecommunication services. To date, there are several generations of PON [1, 2, 3, 4] at speeds up to $10 \mathrm{Gbit} / \mathrm{s}$ on one "PON" -tree. Many works devoted to the problems of the implementation of such networks $[5,6,7,8$, $9,10,11]$, while the problem of balancing the branches of "PON"-trees is solved only for 1:2 splitters type (one input two outputs), for which the corresponding two-dimensional tables $[5,6,7]$ are constructed, and the solution itself is reduced to a simple search from the list of possible values of the coefficients of the division of optical power indicated by the manufacturers of passive optical splitters. For splitters for three or more outputs, such tables do not create at all, because for splitters whith three outputs, these tables become three-dimensional, and for splitters whith four outputs four-dimensional, etc. As a result, the potential of passive optical networks is often neglected in the projected PON, since the networks are become closer to the "star"-topology instead of the "tree" or "bus". At the same time there is no saving of fibers; requirements for automatic gain control systems are increased; the maximum range of "PON"-trees is reduced, which together leads to a reduction in the technical and economic efficiency of PON.

Therefore, the searching of precise analytical solution of balancing passive optical networks problem is an important scientific task, and the technologies of manufacturing of passive optical splitters, which allow to obtain the splitter with the step of dividing the input power at $1 \%$, confirm the relevance of the problem and the finding solutions.

In works $[12,13,14]$ the issues of implementation of passive optical access networks, namely, the balancing of network branches, were considered. In this case, models of passive optical splitters with one input and two and three outputs are proposed. And on the basis of the obtained equations and performed calculations the universal mathematical model (1) was developed.

$\left\{\begin{array}{c}D_{i}=\frac{100}{\sum_{n=1}^{N} 10^{\frac{\Delta_{n} i}{11.5}}} \\ i=1 \cdots N\end{array}\right.$ 
where: $D_{i}$ - percentage of power transmitted from the splitter input in the direction of the output $i$;

$N$ - number of splitter output;

$\Delta_{n i}{ }^{S}$ - difference of insertion loss between $\mathrm{ONT}_{\mathrm{n}}$ and $\mathrm{ONT}_{\mathrm{i}}$, due to the irregular distribution of input power $\mathrm{P}_{\mathrm{IN}}$, which allows balancing of splitter with any number of outgoing ports, and in which as the base approximation formula of the optical splitter losses was proposed in [6] have the form (2):

$$
\begin{aligned}
& A_{S i}=10 \lg \left(\frac{100 \%}{D_{i} \%}\right)+ \\
& +\ln (N-1) \cdot 0.4+0.2+1.5 \lg \left(\frac{100 \%}{D_{i} \%}\right)
\end{aligned}
$$

where: $A_{S i}$ - optical splitters loss towards outputs $i$;

$D_{i}$ - percentage of power transmitted from the splitter input in the direction of the output $i$;

$N$ - number of splitter output.

\section{SETTING UP THE TASK}

In [15] another expression was obtained for the basic approximation formula for optical splitter losses, which depends on the parameter $\beta$, which takes into account the specific type of splitter (3) and two classes of accuracy (Table 1):

$$
A_{S i}=10 \lg \left(\frac{100 \%}{D_{i} \%}\right)+\beta \lg \left(\frac{100 \%}{D_{i} \%}(N-1)\right)
$$

where: $A_{S i}$ - optical splitters loss towards outputs $i, d B$;

$D_{i}$ - percentage of power transmitted from the splitter input in the direction of the output $i, \%$;

$N$ - number of splitter output.

\begin{tabular}{|c|c|c|c|c|c|c|c|c|c|c|}
\hline \multicolumn{2}{|c|}{ Splitter } & $\stackrel{?}{\simeq}$ & ?: & \pm & 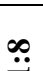 & $\underset{7}{\mathcal{Y}}$ & $\frac{0}{\because}$ & & $\widetilde{?}$ & țִ \\
\hline \multirow[t]{2}{*}{$\beta$} & $\begin{array}{c}\text { Class } \\
\text { A } \\
\Delta \lambda \pm 20 \\
\mathrm{~nm}\end{array}$ & $\stackrel{n}{0}$ & $\begin{array}{l}n \\
\tilde{o}\end{array}$ & $\begin{array}{l}\text { an } \\
\text { on }\end{array}$ & ?. & $\stackrel{n}{n}$ & $\stackrel{n}{n}$ & $\stackrel{n}{0}$ & $\stackrel{t}{0}$ & to \\
\hline & $\begin{array}{c}\text { Class } \\
\text { B } \\
\Delta \lambda \pm 40 \\
\text { nm }\end{array}$ & ก & n & $\stackrel{t}{0}$ & $\begin{array}{l}n \\
n \\
0\end{array}$ & $\stackrel{2}{8}$ & $\hat{0}$ & $\begin{array}{l}\hat{\infty} \\
\infty \\
0\end{array}$ & $\begin{array}{l}\hat{\infty} \\
0 \\
0\end{array}$ & $\stackrel{\infty}{0}$ \\
\hline
\end{tabular}

$\beta$ - correction coefficient (Table 1).

Table 1: The value of the coefficient $\beta$
Thus we formulate the problem in the following form - to investigate the issue of balancing the network branches; to offer a model of passive optical splitters with one input and two and three outputs; and to offer a mathematical model on the basis of the obtained equations and performed calculations that will allow balancing splitters with any number of outgoing ports, in which, as the base approximation formula for optical splitter losses are the expression proposed in [6] and has the form (2).

\section{ANALITICAL MODEL OF THE PASSIVE OPTICAL NETWORKS BALANSING}

\subsection{Balancing PON branches using splitters 1:2}

Consider the model of optical splitter type 1:2 with the scheme of connecting of two remote subscribers, which are represented by the corresponding Optical Network Terminal (ONT): $\mathrm{ONT}_{1}$ and $\mathrm{ONT}_{2}$ (Figure 1).

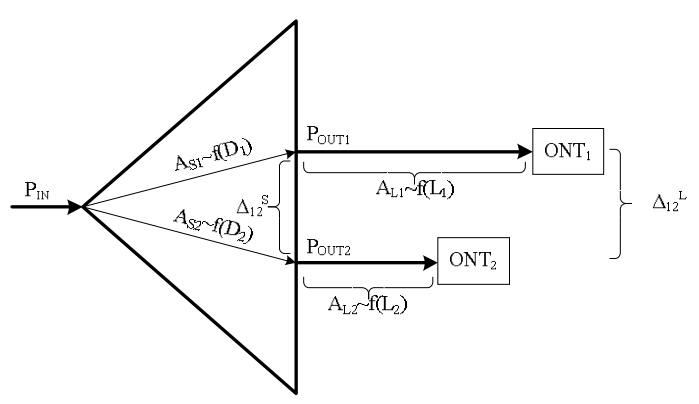

Figure 1: The model of optical splitter type 1:2

In general, subscribers are in different places, that is, the length of individual optical fibers, the number and characteristics of connectors and welding points are different. This leads to the fact that the losses of $A_{\mathrm{Li}} \sim \mathrm{f}\left(\mathrm{L}_{\mathrm{i}}\right)$, which are introduced by individual optical paths in sections from splitter to ONT, are not equal. Therefore, at ONT inputs there is a difference in power levels of optical signals:

$\Delta_{12}^{L}=A_{L 1}-A_{L 2}$

where: $A_{L 1}, A_{L 2}$ - individual total loss of the optical path for $\mathrm{ONT}_{1}$ and $\mathrm{ONT}_{2}$, respectively;

$\Delta_{12}{ }^{L}$ - the difference between power levels of optical signals between $\mathrm{ONT}_{1}$ and $\mathrm{ONT}_{2}$.

To ensure maximum efficiency (range, reliability, stability, error rate) of PON networks, it is necessary to ensure the same level of power at the inputs of all ONTs, which can be done by irregular distribution of power between the outputs of the splitter. From the scheme of Figure 1 it can be seen that the losses introduced by the optical splitter depend on the portion of the power output to the corresponding output, while at the output of the splitter, it is possible to obtain the following 
difference in the losses $\Delta_{12}{ }^{S}$ that will compensate for the inequality of the power levels of the optical signals $\Delta_{12}{ }^{L}$ taken with the negative sign:

$\Delta_{12}{ }^{S}=A_{S 1}-A_{S 2}=-\Delta_{12}{ }^{L}=-\left(A_{L 1}-A_{L 2}\right)=A_{L 2}-A_{L 1}$

where: $A_{S 1}, A_{S 2}$ - optical splitters loss towards outputs $\mathrm{OUT}_{1}$ and $\mathrm{OUT}_{2}$;

$\Delta_{12}{ }^{S}$ - difference of insertion loss between $\mathrm{ONT}_{1}$ and $\mathrm{ONT}_{2}$ due to the irregular distribution of input power $\mathrm{P}_{\mathrm{IN}}$.

The losses introduced by an optical splitter of any type can be approximated by the formula (3), which is converted to the form of expression (2) in the form:

$$
A_{S i}=10 \lg \left(\frac{100 \%}{D_{i} \%}\right)+\lg (N-1) \cdot \beta+\beta \lg \left(\frac{100 \%}{D_{i} \%}\right)
$$

where: $A_{S i}$ - optical splitters loss towards outputs $i$;

$D_{i}$ - percentage of power transmitted from the splitter input in the direction of the output $\mathrm{i}$;

$N$ - number of splitter output.

$\beta$ - correction coefficient (Table 1).

Then, taking into account (4), (5), (6) and the fact that the sum of all $D_{i}$ is equal to $100 \%$, that is:

$$
\sum_{i=1}^{N} D_{i}=100 \%
$$

we can write the following equation system:

$$
\left\{\begin{array}{c}
\Delta_{12}{ }^{S}=A_{L 2}-A_{L 1} \\
D_{1}+D_{2}=100 \\
A_{S 1}=10 \lg \left(\frac{100}{D_{1}}\right)+\lg (2-1) \cdot \beta+\beta \lg \left(\frac{100}{D_{1}}\right) \\
A_{S 2}=10 \lg \left(\frac{100}{D_{2}}\right)+\lg (2-1) \cdot \beta+\beta \lg \left(\frac{100}{D_{2}}\right)
\end{array}\right.
$$

We substitute the third and fourth equations of the system of equations (8) in the first, which will take the form:

$\Delta_{12}{ }^{S}=(10+\beta) \lg \left(\frac{D_{1}}{D_{2}}\right)$

From the second equation of system (8) we express $D_{1}$ and substitute it in (9), we obtain:

$\Delta_{12}{ }^{S}=(10+\beta) \lg \left(\frac{100-D_{2}}{D_{2}}\right)$
The solution (10) for $D_{2}$ has the form:

$D_{2}=\frac{100}{1+10^{\frac{\Delta_{12} S}{(10+\beta)}}}$

Finally for $D_{1}$ and $D_{2}$, we will have:

$$
\left\{\begin{array}{l}
D_{1}=\frac{100}{1+10^{\frac{-\Delta_{12}}{(10+\beta)}}} \\
D_{2}=\frac{100}{1+10^{\frac{\Delta_{12}}{(10+\beta)}}}
\end{array}\right.
$$

Analyzing the system of equations (12), it is possible to simplify its form. So, if the degree indicator $-\Delta_{12} S$ is replaced by $\Delta_{21}{ }^{S}$, where the difference is taken in the opposite direction, and " 1 " in the denominators of the first and second equations of the system (12) are replaced by the corresponding expressions $10^{\frac{4_{1}^{S} S^{5}}{(1+\beta)}}$ and $10^{\frac{\Delta_{2} 2^{S}}{(10+\beta)}}$, we obtain:

$$
\left\{\begin{array}{l}
D_{1}=\frac{100}{10^{\frac{\Delta_{11} s}{(10+\beta)}}+10^{\frac{\Delta_{21} s}{(10+\beta)}}} \\
D_{2}=\frac{100}{10^{\frac{\Delta_{22} s}{(10+\beta)}} 1+10^{\frac{\Delta_{12} s}{(10+\beta)}}}
\end{array}\right.
$$

Next, we will change the order of summation in the second equation of the system (13):

$$
\left\{\begin{array}{l}
D_{1}=\frac{100}{10^{\frac{\Delta_{11}}{(10+\beta)}}+10^{\frac{\Delta_{21}}{(10+\beta)}}} \\
D_{2}=\frac{100}{10^{\frac{\Delta_{12} S}{(10+\beta)}}+10^{\frac{\Delta_{22} S}{(10+\beta)}}}
\end{array}\right.
$$

From which it is clear that the general solution can be finally written as follows:

$$
\left\{\begin{array}{c}
D_{i}=\frac{100}{\sum_{n=1}^{2} 10^{\frac{\Delta_{n i} S}{(10+\beta)}}} \\
i=1 \cdots 2
\end{array}\right.
$$

\subsection{Balancing PON branches using splitters 1:3}

Consider the model of optical splitter type 1:3 (Figure 2) with the scheme of connection of three remote subscribers, which are represented by the corresponding ONT1, ONT2 and ONT3. 


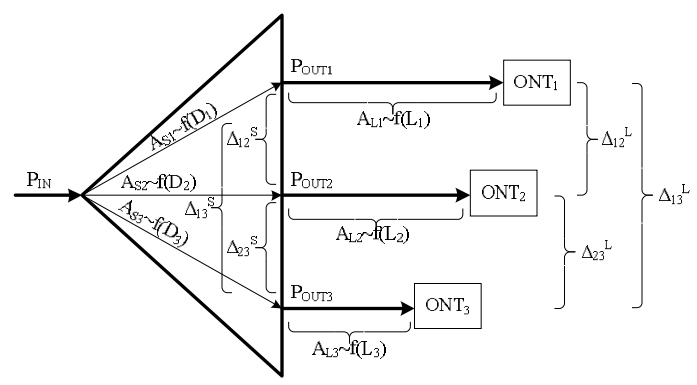

Figure 2: The model of optical splitter type 1:3

In general, subscribers are in different places, so there are three differences in the power levels of optical signals, which we describe as follows:

$\left\{\begin{array}{l}\Delta_{12}{ }^{L}=A_{L 1}-A_{L 2} \\ \Delta_{23}{ }^{L}=A_{L 2}-A_{L 3} \\ \Delta_{13}{ }^{L}=A_{L 1}-A_{L 3}\end{array}\right.$

where: $A_{L 1}, A_{L 2}, A_{L 3}$ - individual total loss of the optical path for $\mathrm{ONT}_{1}, \mathrm{ONT}_{2}$ and $\mathrm{ONT}_{3}$, respectively;

$\Delta_{12}{ }^{L}$ - the difference between power levels of optical signals between $\mathrm{ONT}_{1}$ and $\mathrm{ONT}_{2}$;

$\Delta_{23}{ }^{L}$ - the difference between power levels of optical signals between $\mathrm{ONT}_{2}$ and $\mathrm{ONT}_{3}$;

$\Delta_{13}{ }^{L}$ - the difference between power levels of optical signals between $\mathrm{ONT}_{1}$ and $\mathrm{ONT}_{3}$.

From the scheme of Figure 2 it can be seen that the losses introduced by the optical splitter depend on the portion of the power output to the corresponding output, while at the output of the splitter, it is possible to obtain the following difference in the losses $\Delta_{12}{ }^{S}, \Delta_{23}{ }^{S}, \Delta_{13}{ }^{S}$, that will compensate for the inequality of the power levels of the optical signals $\Delta_{12}{ }^{L}$, $\Delta_{23}{ }^{L}, \Delta_{13}{ }^{L}$, taken with the negative sign:

$\left\{\begin{array}{c}\Delta_{12}{ }^{S}=A_{S 1}-A_{S 2}=-\Delta_{12}{ }^{L}=-\left(A_{L 1}-A_{L 2}\right)=A_{L 2}-A_{L 1} \\ { }^{S}{ }^{S}=A_{S 2}-A_{S 3}=-\Delta_{23}{ }^{L}=-\left(A_{L 2}-A_{L 3}\right)=A_{L 3}-A_{L 2} \\ \Delta_{13}{ }^{S}=A_{S 1}-A_{S 3}=-\Delta_{13}{ }^{L}=-\left(A_{L 1}-A_{L 3}\right)=A_{L 3}-A_{L 1}\end{array}\right.$

where: $A_{S 1}, A_{S 2}, A_{S 3}$ - optical splitters loss towards outputs $\mathrm{OUT}_{1}, \mathrm{OUT}_{2}$ and $\mathrm{OUT}_{3}$ respectively;

$\Delta_{12}{ }^{S}$ - difference of insertion loss between $\mathrm{ONT}_{1}$ and $\mathrm{ONT}_{2}$ due to the irregular distribution of input power $\mathrm{P}_{\mathrm{IN}}$;

$\Delta_{23}{ }^{S}$ - difference of insertion loss between $\mathrm{ONT}_{2}$ and $\mathrm{ONT}_{3}$ due to the irregular distribution of input power $\mathrm{P}_{\mathrm{IN}}$;
$\Delta_{13}{ }^{S}$ - difference of insertion loss between $\mathrm{ONT}_{1}$ and $\mathrm{ONT}_{3}$ due to the irregular distribution of input power $\mathrm{P}_{\mathrm{IN}}$.

Then, taking into account (16), (17), (6) and (7), we can write the following system of equations:

$$
\left\{\begin{array}{c}
\Delta_{12}{ }^{S}=A_{L 2}-A_{L 1} \\
\Delta_{23}{ }^{S}=A_{L 3}-A_{L 2} \\
\Delta_{13}{ }^{S}=A_{L 3}-A_{L 1} \\
D_{1}+D_{2}+D_{3}=100 \\
A_{S 1}=10 \lg \left(\frac{100}{D_{1}}\right)+\lg (3-1) \cdot \beta+\beta \lg \left(\frac{100}{D_{1}}\right) \\
A_{S 2}=10 \lg \left(\frac{100}{D_{2}}\right)+\lg (3-1) \cdot \beta+\beta \lg \left(\frac{100}{D_{2}}\right) \\
A_{S 3}=10 \lg \left(\frac{100}{D_{3}}\right)+\lg (3-1) \cdot \beta+\beta \lg \left(\frac{100}{D_{3}}\right)
\end{array}\right.
$$

Substitute the fifth and sixth equations of the system (18) in the first equation, the sixth and seventh in the second and fifth and seventh in the third and after the transformations we obtain:

$$
\left\{\begin{array}{c}
\Delta_{12}{ }^{S}=(10+\beta) \lg \frac{D_{1}}{D_{2}} \\
\Delta_{23}{ }^{S}=(10+\beta) \lg \frac{D_{2}}{D_{3}} \\
\Delta_{13}{ }^{S}=(10+\beta) \lg \frac{D_{1}}{D_{3}} \\
D_{1}+D_{2}+D_{3}=100 \\
A_{S 1}=10 \lg \left(\frac{100}{D_{1}}\right)+\lg (3-1) \cdot \beta+\beta \lg \left(\frac{100}{D_{1}}\right) \\
A_{S 2}=10 \lg \left(\frac{100}{D_{2}}\right)+\lg (3-1) \cdot \beta+\beta \lg g\left(\frac{100}{D_{2}}\right) \\
A_{S 3}=10 \lg \left(\frac{100}{D_{3}}\right)+\lg (3-1) \cdot \beta+\beta \lg \left(\frac{100}{D_{3}}\right)
\end{array}\right.
$$

Having solved this equation for $D_{1}, D_{2}, D_{3}$, we obtain:

$$
\left\{\begin{array}{l}
D_{1}=\frac{100}{1+10^{\frac{-\Delta_{12} s}{(10+\beta)}}+10^{\frac{-\Delta_{13} s}{(10+\beta)}}} \\
D_{2}=\frac{100}{1+10^{\frac{\Delta_{12} s}{(10+\beta)}}+10^{\frac{-\Delta_{23} s}{(10+\beta)}}} \\
D_{3}=\frac{100}{1+10^{\frac{\Delta_{13} s}{(10+\beta)}}+10^{\frac{\Delta_{23} s}{(10+\beta)}}}
\end{array}\right.
$$

Analyzing the expression (20) it is possible to simplify it form. Thus, if the exponent $-\Delta_{12}{ }^{S},-\Delta_{13}{ }^{S},-\Delta_{23}{ }^{S}$ substitute by $\Delta_{21}{ }^{s}, \Delta_{31}{ }^{S}, \Delta_{32}{ }^{S}$, where the difference is taken in the opposite direction and one's in the denominator 
of the first, second and third equation of (18) replace by the

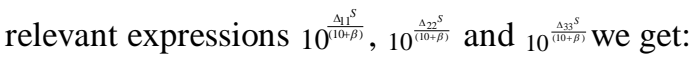

$$
\left\{\begin{array}{l}
D_{1}=\frac{100}{10^{\frac{\Delta_{11} s}{(10+\beta)}}+10^{\frac{\Delta_{21} s}{(10+\beta)}}+10^{\frac{\Delta_{31} s}{(10+\beta)}}} \\
D_{2}=\frac{100}{10^{\frac{\Delta_{22} s}{(10+\beta)}}+10^{\frac{\Delta_{12} s}{(10+\beta)}}+10^{\frac{\Delta_{32} s}{(10+\beta)}}} \\
D_{3}=\frac{100}{10^{\frac{\Delta_{33} s}{(10+\beta)}}+10^{\frac{\Delta_{13} s}{(10+\beta)}}+10^{\frac{\Delta_{23} s}{(10+\beta)}}}
\end{array}\right.
$$

Further, changing the order of summation in the second and third equations of system (21), similar to the formula (15), finally we get a compact form of solution for determine the rate of distribution of power between the outputs of splitters 1:3 for precise balancing of PON branches:

$$
\left\{\begin{array}{c}
D_{i}=\frac{100}{\sum_{n=1}^{3} 10^{\frac{\Delta_{n i}}{(10+\beta)}}} \\
i=1 \cdots 3
\end{array}\right.
$$

\subsection{Balancing equation of PON branches by splitters of general type 1:N}

Consider the model of optical splitter type 1:N with the scheme of connecting of $N$ remote subscribers (Figure 3 ).

Comparing (15) and (22) shows that they have the same form exactly the number of outputs of optical splitter, that is the type of splitter.

Thus it can be argued that for precise balancing of optical splitters 1:N (Figure 3), where $\mathrm{N}$ is a positive integer and is equal to the number of outputs, can be used one compact general formula:

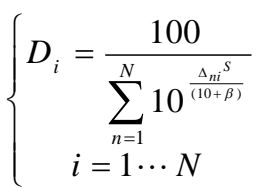

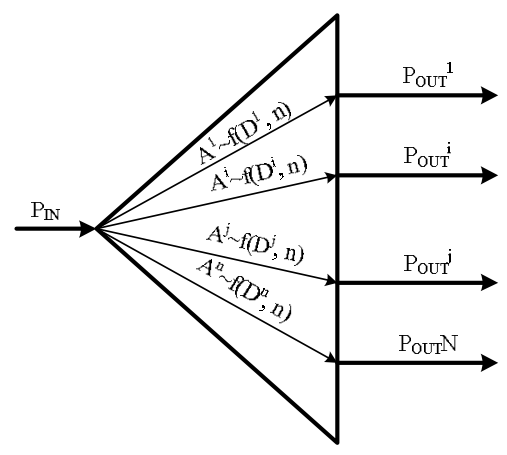

Figure 3: The model of optical splitter type 1:N
Thus, an analytical expression for balancing passive optical splitters (23) is obtained, which allows to carry out the balancing of splitter with any number of output ports, in which the expression proposed in [6] is the base formula for approximation of losses by an optical splitter is obtained. Comparing expressions (1) and (23), it is evident that they are of the same form and differ only in the value of the denominator of the power index in the sum that is in the denominator of the general expression. In formula (1), this value has a constant value of 11.5 , and in formula (23) it varies depending on the parameter $\beta$, which takes into account the particular type of splitter (3) and two classes of accuracy (Table 1).

So, the obtained results are ready for use in Automatic design systems like [16] for passive optical networks as an infrastructure component of Internet of Things and 5G [17].

\section{CONCLUSION}

1. A mathematical model of passive optical networks of a complex structure is developed, which can be used both in the implementation of passive optical access networks, and in cable television networks where the physical processes in the couplers and splitters are very similar.

2. Due to the use of the analytical solution of the balancing problem, it became possible in practice to approach the theoretical values embodied in PON technology.

3. The resulting analytical expression for balancing passive optical splitters has a uniform form and differs only with the value of the denominator of the power index in the sum that is in the denominator of the general expression and depends on the parameter $\beta$ which takes into account the particular type of the splitter and the two classes of accuracy.

4. The obtained results are ready for use in Automatic design systems for passive optical networks based on $\beta$-parametric approximation formula.

\section{REFERENCES}

1. ITU-T Rec. G.983.1, Broadband Optical Access Systems based on Passive Optical Networks (PON), 10/1998.

2. ITU-T Rec. G.983.3, A broadband optical access system with increased service capability by wavelength allocation, 03/2001.

3. ITU-T Rec. G.984.3, Gigabit Capable Passive Optical Networks (G-PON): Transmission Convergence Layer Specification, 10/2003.

4. ITU-T Rec. G.987, 10-Gigabit-capable passive optical network(XG-PON) systems: Definitions, abbreviations and acronyms, 06/2012.

5. D.B. Glushchenko, V.N. Uryadov. Methods for estimating the budget based on the application of technical solutions using a component of passive optical networks, Communications Networks, No. 2 (108), pp. 43-45, 2011. 
6. D.B. Glushchenko, V.N. Uryadov. PON optical budget calculation with ununiform splitters, in Modern means of communication: materials XV Intern. Scientific-techn. Conf, Minsk, Republic of Rep. Belarus. VGKS, 28-30 Sept. 2010, p.29.

7. D.B. Glushchenko, V.N. Uryadov. PON optical budget calculation with uniform splitter, in Modern means of communication: materials XV Intern. Scientific-techn. Conf, Minsk, Republic of Rep. Belarus. VGKS, 28-30 Sept. 2010, p.28.

8. I.I. Petrenko, R.R. Ubaidullaev. Passive Optical Networks PON, Part 1. Architecture and Standards, Lightwave Russian edition, No. 1, pp. 22-28, 2004.

9. E.A. Rusakova. Designing of access network base on PON technology, Ekaterinburg, Russia, 2015, pp. 40.

10. A.K. Sklyarov. Optical fiber network and communication systems, St. Petersburg, Russia, 2010, pp. 272.

11. R.R. Ubaidullaev. Fiber-optic networks, Eco-Trends, Moscow, Russia 2001, pp. 267.

12. O.L. Nedashkivskiy. Precise method of balancing passive optical networks with irregular splitter with two or more outputs, IEEE Xplore 4-7 July 2017, pp. 228-231.(https://ieeexplore.ieee.org/document/802010)

13. O. Nedashkivskiy. Precise method of balancing passive optical networks with irregular splitter with two or more outputs, in 2017 2nd International Conference on Advanced Information and Communication Technologies (AICT), Lviv. Ukraine. - 4-7 July 2017, pp. 228-231, doi: 10.1109/AIACT.2017.8020107.

14. O.L. Nedashkivskiy, A.I. Semenko. Precise method of balancing passive optical networks with irregular splitter with two or more outputs, Meridian Ingineresc. Journal of Technical University of Moldova and Moldavian Engineering Association, №2, pp. 18-21, 2017.

15. V.I. Yefanov, Ya.S.Grinshpon, D.V. Vodzhayev, A.S. Utkina. Calculation of losses in splitter of optical power and attenuation in an optical fiber, T-COMM Telecommunications and transport, Vol. 8, pp. 39-41, 2014.

16. Dalila B.M.M. Fontes, Paulo A. Pereira, Fernando A.C.C. Fontes. A Decision Support System for TV self-promotion Scheduling. International Journal of Advanced Trends in Computer Science and Engineering, vol. $\quad 8, \quad$ No.2, pp. 134-140, 2019. https://doi.org/10.30534/ijatcse/2019/06822019.

17. Radhia Khdhir, Aymen Belghith. 5G LTE-A Cognitive Multiclass Scheduling Scheme for Internet of Things. International Journal of Advanced Trends in Computer Science and Engineering, vol. 8, No.5, pp. 2485-2491, 2019. https://doi.org/10.30534/ijatcse/2019/94852019. 